\title{
Mechanisms of Switching Response to External Phosphate Levels in Escheria coli
}

\author{
Cansu Uluşeker ${ }^{1,3}$, Martin M. Hanczyc ${ }^{1,4}$, Ozan Kahramanoğulları ${ }^{2}$ \\ ${ }^{1}$ Centre for Integrative Biology, University of Trento, Trento, Italy \\ ${ }^{2}$ Department of Mathematics, University of Trento, Trento, Italy \\ ${ }^{3}$ The Microsoft Research - University of Trento Centre for Computational and Systems Biology, Rovereto, Italy \\ ${ }^{4}$ Chemical and Biological Engineering, University of New Mexico, Albuquerque USA \\ ozan.kahramanogullari@unitn.it
}

\begin{abstract}
The phosphate economy in cells is essential in many biochemical processes from signal transduction, to energy metabolism to DNA and RNA synthesis. All living systems therefore acquire and regulate phosphate in order to survive and reproduce. E. coli, for example, regulate the inorganic phosphate $\left(P_{i}\right)$ uptake in order to survive under phosphatelimiting conditions. To achieve this, E. coli have developed an accurate control mechanism, Pho regulon, to adapt to environmental perturbations of $P_{i}$, controlled by the PhoR/PhoB two-component regulatory system (TCS). The signalling of the TCS is delivered by interactions with the ABC transporter via PhoU. However, the exact mechanisms of interaction are unknown. Here, we propose mechanistic explanations for these mechanisms via a quantitative computational analysis, whereby we model plausible ABC and TCS state transitions. We analyse the interaction mechanism and the dynamic behaviour of TCS system deactivation in relation to the external $P_{i}$ levels. We show that the behaviour of this system depends on the network structure. In particular, we use alternative models to demonstrate that variation in interaction patterns affect the response time of the system. Overall we show how to model a system where some key interactions are as yet unknown and to provide testable predictions that can easily be verified in the lab. This way, modelling is being used to increase our mechanistic understanding of important biological systems by defining and driving wet lab experiments and to increase our biological understanding of the often complex relationship between an organism and its environment.
\end{abstract}

\section{Introduction}

Living cells, like bacteria, face a wide range of challenging environmental conditions such as nutrient limitation or exposure to antibiotics. Therefore, they must sense and rapidly produce the appropriate response to their environment. Response networks of $E$. coli are selected for fast and reliable adaptation to environmental conditions (Celani and Vergassola, 2009). A mechanism that governs these responses is given by the two-component systems (TCSs) (Shimizu, 2014). TCSs transmit information between a histidine kinase (HK) sensor and a cognate response regulator (RR) by phosphorylation (Shimizu, 2014). A particularly wellcharacterised example of a TCS histidine kinase is PhoR in
E. coli. PhoR responds to the changes in the external inorganic phosphate $\left(P_{i}\right)$ level and controls the phosphorylation of the response regulator PhoB (Wanner, 1996; SantosBeneit, 2015). The phosphorous compounds are essential nutrients for many biomolecules and have important roles in cell function and life (Wanner, 1996). Therefore, through such metabolic architectures, proper $P_{i}$ signaling produces robust growth of E. coli.

Many parts of this $P_{i}$ signaling pathway are known. $E$. coli control $P_{i}$ metabolism through Pho regulon (Wanner, 1996; Santos-Beneit, 2015; Lamarche et al., 2008). Pho regulon, a well studied system in E. coli, is a global regulatory circuit involved in $P_{i}$ management (Wanner, 1996; SantosBeneit, 2015; Lamarche et al., 2008; Chekabab et al., 1993; Wanner et al., 1995; Gao and Stock, 2009). It is central to assimilation of $P_{i}$ and regulation of $P_{i}$ metabolism. It includes a number of $P_{i}$ starvation-inducible genes such as (Wanner, 1996; Wanner et al., 1995)

1. two that are members of the large family of two component systems (TCSs), a sensor histidine kinase PhoR and a response regulator PhoB;

2. four components of the $\mathrm{ABC}$ transporter Pst (PstSCAB) which are an extracellular binding protein (PstS), two transmembrane proteins (PstC, PstA) that form the transmembrane domain (TMD), and a dimer of cytosolic peripheral proteins (PstB) referred to as the nucleotidebinding domain (NBD);

3. and the chaperone-like $\mathrm{PhoR} / \mathrm{PhoB}$ inhibitory protein called PhoU.

Figure 1 displays a schematic representation of the Pho regulon system. Limiting concentrations of extracellular $P_{i}$ activates the system, resulting in the phosphorylation of transcription factor PhoB by PhoR (Wanner, 1996; SantosBeneit, 2015; Chekabab et al., 1993). Signal transmission occurs through autophosphorylation of PhoR, followed by transfer of the phosphoryl group to PhoB (Wanner, 1996; Santos-Beneit, 2015; Lamarche et al., 2008; Chekabab et al., 1993; Wanner et al., 1995). Phosphorylated PhoB activates Pho regulon by binding to a consensus DNA sequence 
within the promoters of Pho regulon genes (Wanner, 1996; Santos-Beneit, 2015; Lamarche et al., 2008; Chekabab et al., 1993; Wanner et al., 1995).

A return to the repression state occurs by a transition from the growth state to a phosphate-rich environment. The activation signal is interrupted by PhoR acting as a phosphatase on phosphorylated PhoB. System deactivation upon stimulus removal has an important task of coordinating and regulating the cell growth (Wanner, 1996; Santos-Beneit, 2015; Lamarche et al., 2008; Chekabab et al., 1993; Wanner et al., 1995). Although, there has been much research on activation dynamics of TCS (Shinar et al., 2007; Gao and Stock, 2009), it is not well understood how PhoR receives the signal from PhoU. It is known that, under environmental $P_{i}$ repleted conditions, the Pho regulon is not induced (off state) and $\mathrm{PhoB}$ is maintained in the non-phosphorylated form by PhoR (Lamarche et al., 2008; Chekabab et al., 1993).

It has been suggested that the PhoR/PhoB proteins assess $P_{i}$ availability by monitoring the activity of the ABC transporter. In addition to the $\mathrm{ABC}$ transporter, $\mathrm{PhoU}$ is also required for $P_{i}$ signal transduction (Wanner, 1996; Gardner et al., 2014; Rice et al., 2009). PhoU constitutes an intermediate step between the ABC and TCS systems, inhibiting PhoR when the ABC system actively transports $P_{i}$ (Wanner, 1996; Gardner et al., 2014; Rice et al., 2009). When PhoU is mutated or deleted, PhoR continuously phosphorylates PhoB (Gardner et al., 2014). Then, phosphorylated PhoB delivers the high expression of the Pho regulon genes. $\mathrm{PhoU}$ is involved not only in the control of autokinase activity, but also in control of $\mathrm{ABC}$ transporter in order to avoid an uncontrolled $P_{i}$ uptake. Moreover, it is known that PhoU and $\mathrm{ABC}$ transporter are also required for dephosphorylation of phospho-PhoB (Wanner, 1996; Wanner et al., 1995). However, the exact mechanism of PhoU action is not fully elucidated. Here, we address these uncertainties by applying alternative models based on plausible interaction patterns. This allows us to predict the unknown mechanisms of the system, which can be verified by experiments.

In order to address these questions, we have analysed the signal transfer pattern from the ABC transporter to the histidine kinase PhoR under the conditions of various external $P_{i}$ concentrations. For this, we have designed alternative models to quantify and evaluate the events of the deactivation mechanism in the system. The main contribution of our model is thus the mechanistic quantification of the continuous relationship between the external $P_{i}$ concentration and the PhoR activity in the cell, delivered by the signal transduction mechanism through $\mathrm{ABC}$ transporter and PhoU. This, in return, provides predictions on the effects of the $\mathrm{ABC}$ transporter and $\mathrm{PhoU}$ to the system deactivation.

In the following, we distinguish different types of interactions, and show how these patterns can correspond to signalling mechanisms. For this, we have first implemented our models in terms of hypothetical direct interactions between
$\mathrm{ABC}$ transporter and PhoR. Thus, our preliminary model includes an implicit representation of the PhoU effect on the system. We have then extended and refined this implicit representation with an explicit mechanistic characterisation of PhoU that supports the preliminary observations. We have performed a computational analysis by simulations to enhance the understanding of the effects of multiple pathways on response dynamics and protein homeostasis in different environmental conditions. Our simulations with various mechanisms provide predictions on the effect of the system structure and PhoU activity. In particular, we have analysed how strong phosphatase activity suppresses TCS activation, and quantified how fast the system is turned off once the stimulus due to external $P_{i}$ concentration is removed.

\section{Methods}

In a system of $E$. coli, it is important to balance $P_{i}$ accumulation (Wanner, 1996; Lamarche et al., 2008; Wanner et al., 1995). When external $P_{i}$ levels are low, it becomes a growth limiting factor (Peterson et al., 2005). Therefore, the cell invests resources to produce proteins, rather than $P_{i}$ accumulation (Peterson et al., 2005; Hudek et al., 2016). However, when the external $P_{i}$ is in excess, the E. coli begins to enhance $P_{i}$ accumulation and switches the TCS off. To explain this system, we have previously designed and analysed a two component system model that quantifies the dynamic mechanisms of auto-regulation in E. coli, and explored and verified emerging phenotypes with synthetic promoters (Uluşeker et al., 2018, 2017). Moreover, we have analysed the response of the model to variations in the external $P_{i}$ levels.

In the following, to better illustrate the response of Pho regulon and TCS signalling to the different external $P_{i}$ levels, we present a model as a conceptual representation of switching of TCS. We have considered a set of reactions for the signal of external $P_{i}$ with the TCS histidine kinase as the target for delivery. As a preliminary step, the conceptual model contains implicit representation of the interactions with the TCS. We describe the formation of repression complex, which involves PhoR and $\mathrm{ABC}$ transporter, together with an implicit effect of PhoU protein. The model is consistent with observations in the literature, and maintains accurate relationships between all the components in the system. This enables the model to demonstrate the effect of PhoU protein and its integration to the system.

From an alternative perspective, Figure 2 shows how the regulatory system can be represented as a logic gate. TCS can be viewed in terms of its inputs and outputs without details of internal workings. Thus, it alllows us to implement the role of PhoU implicity. With the help of schematic and logic gate representation of the system we have built a preliminary model to analyze how the signal for external changes is delivered to TCS, especially to PhoR. The signal transduction from PhoR to gene regulation via TCS 
Activation

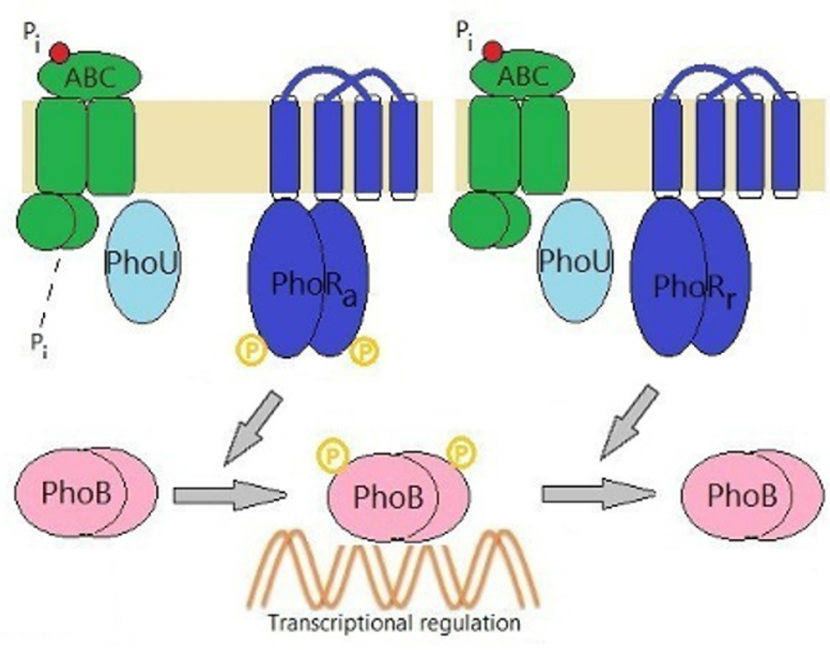

Figure 1: Schematic representation of the different stages of Pho regulon. Green colored shape represents ABC transporter, dark blue one is PhoR, light blue shape stands for PhoU and pink stands for PhoB. The signaling processes of activation and deactivation correspond to different states of PhoR.

Activation: When external inorganic phosphate $\left(P_{i}\right)$ is depleted, the system is in growth condition. Under this condition, Pho regulon is active and produces its components. $\mathrm{ABC}$ transporter binds the external $P_{i}$, internalises and releases it to the cytosol. According to the current biological model in the literature, PhoR assesses $P_{i}$ availability by monitoring the $\mathrm{ABC}$ transporter. This is done by relaying the signal via PhoU. When the system is active, PhoU does not stabilize PhoR and PhoR passes to the active state $\left(P h o R_{a}\right)$. PhoR $R_{a}$ phosphorylates PhoB. Phosphorylated PhoB then acts as a transcription factor for the operon.

Deactivation: PhoR acts as a phosphatase on phosphorylated PhoB. When environmental $P_{i}$ is in excess, Pho regulon is inhibited. PhoU stabilizes PhoR. This prevents PhoR from autophosphorylating itself. The signal is thus propagated to PhoR, resulting in its inhibition state $P h o R_{r}$.

has been quantified in our previous work (Uluşeker et al., 2018, 2017). In the current work, this framework is used as a basis to describe mechanistically the effect of external $P_{i}$ concentration change and TCS inhibition. The model is based on a mechanistic description of the system dynamics within a chemical reaction system representation with respect to mass action dynamics.

$\mathrm{ABC}$ transporter regulates the translocation of $P_{i}$ to the cytosol. $\mathrm{ABC}$ transporter autophosphorylates itself and switches between the closed $(\mathrm{ABC})$ and open $(\mathrm{ABCo})$ dimer

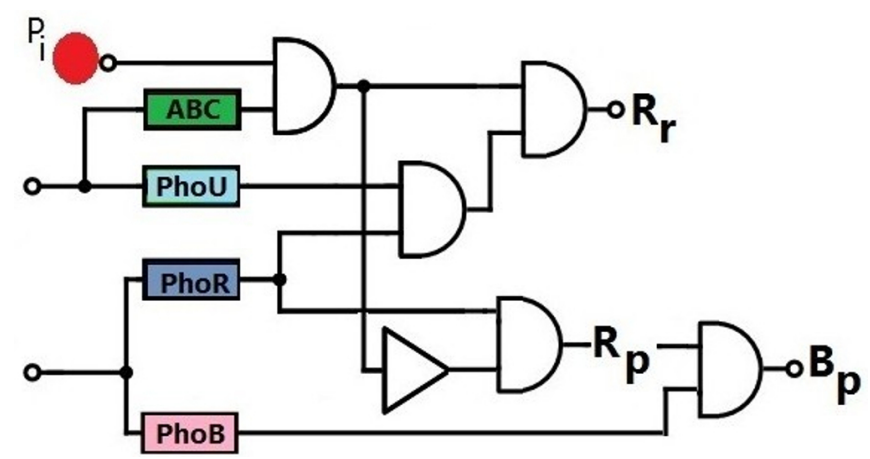

Figure 2: Representation of Pho regulatory system interactions as a logic gate circuit. Triangle shape represents NOT gate, and half circle shapes are AND gates. System inputs are $P_{i}, \mathrm{ABC}, \mathrm{PhoU}, \mathrm{PhoR}$ and PhoB. Colors are used as described in Figure 1. For a 2-input AND gate, the output is true if both inputs are true. While, for a single input NOT gate, the output is only true when the input is false due to the NOT gate. PhoR assesses $P_{i}$ availability by monitoring the activity of the $\mathrm{ABC}$ transporter. When the $P_{i}$ influx is reduced, PhoU unblocks PhoR. This allows PhoR to autphosphorylate itself $\left(R_{p}\right)$ and phosphorylate $\mathrm{PhoB}\left(B_{p}\right)$. Otherwise, when the $P_{i}$ influx is increased, PhoU stabilizes PhoR and prevents PhoR dimers from autphosphorylating, and PhoR becomes repressive $\left(R_{r}\right)$.

conformations as a result of ATP binding. External $P_{i}$ (Pext) intake takes place with the conformational changes in the $\mathrm{ABC}$ transporter. This causes an increase in the amount of internal $P_{i}$ (Wanner, 1996; Hsieh and Wanner, 2010).

$$
\begin{aligned}
& \mathrm{ABC} \longrightarrow \mathrm{ABCo} \\
& \mathrm{ABCo}+\mathrm{Pext} \longrightarrow \mathrm{Pin}+\mathrm{ABC}
\end{aligned}
$$

$\mathrm{PhoU}$ is an essential protein for the repression of the Pho regulon at high $P_{i}$ conditions (Gardner et al., 2014; Wanner, 1996). Although the mechanism behind the Pho regulon deactivation is unknown, one hypothesis is that PhoU inhibits the activation of PhoR by interacting with PhoR and ABC, and converts PhoR to the represive state (PhoRr) (Gardner et al., 2014; Steed and Wanner, 2014; Rice et al., 2009). It is assumed that the $\mathrm{ABC}$ transporter senses the $P_{i}$ levels and transfers the signal to the TCS via PhoU (Gardner et al., 2014).

Therefore, as an initial step of the model component interactions, we have expressed the inhibition as a direct interaction between $\mathrm{ABC}$ and PhoR. We have then constructed two alternative models for for assessing interactions between the $\mathrm{ABC}$ transporter and TCS with potentially distinct mechanisms for diminished PhoR activities. These two alternative models are abstractions that contain PhoU implicitly, which 
we refine below. Our motivation behind the use of alternative reaction designs is to examine how the type of the chemical reactions impacts the switch dynamics.

First possible interaction is:

$$
\begin{aligned}
& \mathrm{ABC}+\mathrm{PhoR} \longrightarrow \mathrm{ABC}+\mathrm{PhoRr} \\
& \mathrm{PhoRr} \longrightarrow \mathrm{PhoR}
\end{aligned}
$$

and other one is expressed as:

$$
\begin{aligned}
& \mathrm{ABC}+\mathrm{PhoR} \longrightarrow \mathrm{ABC} \text { _PhoR } \\
& \mathrm{ABC} \text { _PhoR } \longrightarrow \mathrm{ABC}+\mathrm{PhoR}
\end{aligned}
$$

For these two alternative models, we have implemented the deterministic ordinary differential equation (ODE) systems in Matlab by using the standard translation from the chemical reaction network above, based on stoichiometries. The models have been tested with 0,50 , and $100 \mu \mathrm{M}$ external $P_{i}$. The concentrations of proteins PhoR and $\mathrm{ABC}$ are set to $0.22 \mu \mathrm{M}$ based on literature and verified by our previous work (Van Dien and Keasling, 1997; Uluşeker et al., 2018, 2017). The parameters for the rates are listed in Table 1 . The rate of reaction 1 is obtained in accordance with our previous works (Uluşeker et al., 2018, 2017). The unknown rates of reaction 2 and 3 have been estimated to reproduce decreasing dynamics of the PhoR according to the external $P_{i}$ concentration change with respect to the switching of dynamics. The rate of reverse reaction 4 has been set to the value that delivers the expected dynamics. We have used these values as a calibration for the control models and explored the possible variations as described below.

\begin{tabular}{|c|c|c|c|}
\hline Reaction N. & Rate Symbol & Value & Unit \\
\hline \hline 1 & $\mathrm{r} 1$ & 25.3658 & $\mu M^{-1} \mathrm{sec}^{-1}$ \\
\hline 2 & $\mathrm{r} 2$ & 0.001 & $\mathrm{sec}^{-1}$ \\
\hline 3a and 3b & $\mathrm{r} 3 \mathrm{a}$ and $\mathrm{r} 3 \mathrm{~b}$ & 1 & $\mu M^{-1} \mathrm{sec}^{-1}$ \\
\hline 4a and 4b & $\mathrm{r} 4 \mathrm{a}$ and $\mathrm{r} 4 \mathrm{~b}$ & 0.000001 & $\mathrm{sec}^{-1}$ \\
\hline
\end{tabular}

Table 1: The reaction rates and units of the control model.

\section{Results}

Figure 3 shows a dynamic representation of the two different models used in our analysis. Model 1 includes reactions 1, 2, 3a and 4a and Model 2 includes the reactions 1, 2, 3b and $4 \mathrm{~b}$. The models describe two possible mechanisms for switching off the TCS in response to the $P_{i}$ repletion. The deterministic ODE simulations display the average dynamic behaviour of the concentrations for the simulated 4.5 hours. In the two models, the parameter values of all the processes are the same. This guarantees that the differences in dynamics are only due to the types of the interactions.
The $\mathrm{ABC}$ transporter can act as an enzyme (Model 1) or binds the histidine kinase PhoR (Model 2) in response to an external signal. Both forms of $\mathrm{ABC}$ transporter mechanisms affect the TCS with similar affinities. Both models examine the functionality of the system without going into the details of its internal structures with respect to PhoU. When ABC acts as an enzyme as in Model 1, it indirectly affects PhoR and inhibits PhoR activation while being preserved. In the case of Model 2, ABC participates directly and binds the unphosphorylated PhoR to form a complex (ABC_PhoR). This complex formation causes the stabilisation of PhoR. The stabilised PhoR does not modulate the phosphorylation and activity of relevant proteins. The designed interaction of the regulatory system functions as expected in response to various external $P_{i}$ levels.

The plots clearly show that when the external $P_{i}$ level increases, activity of PhoR decreases in both models (Figure 3). Moreover, the system is able to maintain PhoR activity, thus protein synthesis, to maximal when external $P_{i}$ is 0 $\mu \mathrm{M}$. Figure 3 provides a comparison of the functional effectiveness of the two mechanisms: Model 1 has faster response time than Model 2 for all the external $P_{i}$ levels (Figure 3). Response time is the time to reach halfway between the initial and final levels in the dynamic process (Alon, 2007). It is an important measure for the speed at which PhoR levels change as it quantifies the effectiveness, especially in fluctuating environmental conditions.

To better understand the TCS switching dynamics, we have performed a mathematically controlled comparison of the two models (Alves and Savageau, 2000). We have set the system as equivalent as possible by reaction rates, external conditions and initial values. This allows us to observe the differences in the physiological behaviour between designs. The comparison analysis was done using the specific set of parameter values reported in Table 1. By exploring the effect of the changes in the reaction rates $\mathrm{r} 1, \mathrm{r} 2$, and $\mathrm{r} 3 \mathrm{a}$ and $\mathrm{r} 3 \mathrm{~b}$ in orders of magnitude, we have classified the similarity in dynamics with possible variations in the rates.

Next, we have examined the response time and PhoR degradation for alternative models with the selected parameters. We have extended our calculations and reproduced the dynamics in Figure 4a and Figure 4b. Steady-state levels of PhoR and response time have been cross-examined at reaction rates $r 1, r 2$, and $r 3$, allowing evaluation of the stability of the system with different rates. We have omitted the reaction rate $\mathrm{r} 4$ variation because variations on this rate at this regime imply a departure from the expected dynamics. However, variations on $\mathrm{r} 4$ together with others preserve the dynamics, as we explore below.

Robust adaptation is a biological behaviour for E. coli that describes surviving in varying environmental conditions. We have performed an analysis to predict the robustness of network against variations of its parameters. We have observed that the model preserves the expected dy- 


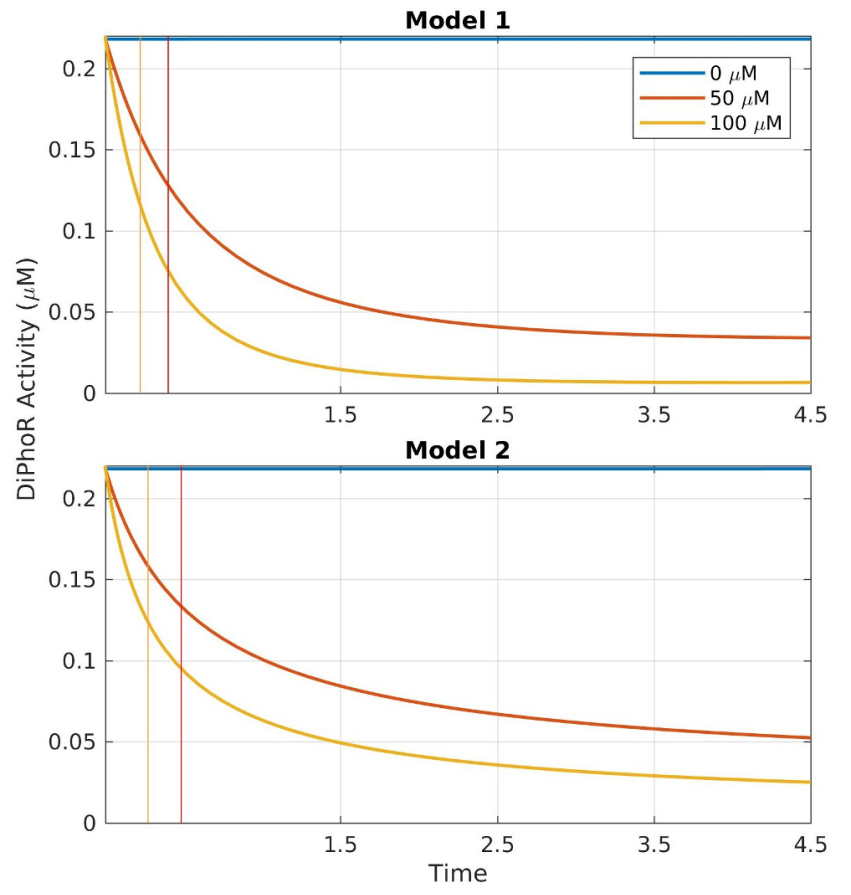

Figure 3: The response of the model to variations in the external $P_{i}$ levels. Model 1 includes the reactions $1,2,3 a$, and $4 a$ and Model 2 includes 1, 2, 3b, and $4 b$. The simulations are performed for the external $P_{i}$ concentrations of 0,50 , and $100 \mu \mathrm{M}$ and a time-course of 4.5 hours is considered. PhoR activity is plotted in blue when $P_{i}$ concentrations is $0 \mu \mathrm{M}$, it is represented with red when external $P_{i}$ concentrations is $50 \mu \mathrm{M}$, and orange color is used when $P_{i}$ concentrations is $100 \mu \mathrm{M}$. Response times are represented with vertical lines. Red line is the response time when the external $P_{i}$ concentration is $50 \mu \mathrm{M}$ and the orange one is for the external $P_{i}$ concentration of $100 \mu \mathrm{M}$.

namics under perturbations of system parameters. That is, changes on rates result in robust adaptation of Pho regulon system. With variations on rates in Model 1, the switch from PhoR to repressed state occurs more quickly than in Model 2 (see Figure 4a and Figure 4b ). As in Figure 4a and Figure $4 \mathrm{~b}$, a scaling of the system rates leads to a time scaling of the output response. The steady state levels are lower in the Model 2 system. Moreover, Figure $4 \mathrm{a}$ and Figure $4 \mathrm{~b}$ demonstrates the role of the conformational change of $\mathrm{ABC}$ on the switch off dynamics. Lower autophosphorylation rate of $\mathrm{ABC}$ results in higher levels of PhoR repression.

The response of the Pho regulon has to be rapid enough for TCS to be switched off as the $P_{i}$ level rises. In the models under comparison, the main difference is in the interaction of $\mathrm{ABC}$ and PhoR. In this situation, the response time of Model 1 is faster than Model 2 (see Figure 4a and Figure $4 b$ ). This comparison is thus relevant for understanding the differences in the dynamic behaviours that are intrinsic to the differences in design. The present analysis of the TCS switch off system thus provides a refined estimation of the phosphatase activity of PhoR for these models. The analysis here on Model 1 and 2 is used below to discuss the PhoU mechanism. Moreover, these models can be extended and refined to analyze the switch off mechanism of the other TCSs in E. coli.

Figure 5 and Figure 6 display the results of the analysis, whereby we have scanned the parameter values for $\mathrm{r} 4 \mathrm{a}$ and $\mathrm{r} 4 \mathrm{~b}$ in orders of magnitude. We have increased the value for the reaction rate, $\mathrm{r} 4 \mathrm{a}$ and $\mathrm{r} 4 \mathrm{~b}$ while keeping the reaction rates $r 3 a$ and $r 3 b$ the same. We have scanned $r 1$ and $r 2$ values by orders of magnitude to observe the variations in system dynamics in Model 1 and 2. We have calculated for each set of reaction rate values the functional effectiveness with respect to PhoR level change and the response time. We have found that if the chosen reaction rate 2 is reduced, when the rate of reaction 4 is increased, then robust adaptation of dynamics is preserved. Moreover, when $\mathrm{r} 4$ is increased, the system has slower response time and lower PhoR level change.

We have analysed the evolution of the E. coli in response to different external $P_{i}$ concentrations. The analysis on two different models with variations in model parameters demonstrate the gain of the system under a broad spectrum of circumstances, represented by these parameters. We have observed that Model 1 has faster response time in all regimes. With the assumption that faster response is more favourable, these results suggest that the enzymatic interaction in Model 1 can be a prevalent regulatory pattern in biological signalling pathways.

\section{Discussion}

Pho regulon expresses many genes that are influenced by the environmental $P_{i}$ level, and their expression is regulated by TCSs. TCS is thus a predominant form of signal transduction in E. coli.

Our results provide a quantitative description of how different proteins interact when the TCS is switched off as a result of the changes in external $P_{i}$ concentration. The effect of the switch off mechanism is measured in terms of PhoR level change. Our results do not only provide predictions on the physiology of the Pho regulon, but also demonstrate how a strong signal due to phosphatase activity can cause a fast switch off response. The comparison of the effect of possible interaction types in our models becomes instrumental for understanding the differences in behaviour of biological circuits created by using synthetic biology techniques. Moreover, this work provides a framework to quantitatively analyse the interactions of PhoU with other system compartments, for example, as in Uluşeker et al. (2018, 2017).

To elucidate the mechanistic function of PhoU, we refine our models with explicit mechanism of PhoU action 
(a)

\begin{tabular}{|c|c|c|c|c|c|c|}
\hline \multirow[t]{3}{*}{$\mathbf{M}$} & \multicolumn{6}{|c|}{ Response Time (sec) } \\
\hline & \multicolumn{2}{|c|}{ r1 } & \multicolumn{2}{|c|}{ r2 } & \multicolumn{2}{|c|}{ r3a } \\
\hline & $50 \mu \mathrm{M}$ & $100 \mu \mathrm{m}$ & $50 \mu \mathrm{M}$ & $100 \mu \mathrm{M}$ & $50 \mu \mathrm{M}$ & $100 \mu \mathrm{M}$ \\
\hline 2 & 0 & 0 & 18 & 12 & 0,05 & 0,05 \\
\hline 1 & 2608 & 2608 & 151 & 85 & 136 & 67 \\
\hline 0 & 1442 & 800 & 1442 & 800 & 1442 & 800 \\
\hline-1 & 164,5 & 80 & 18450 & 7912 & 2264 & 2264 \\
\hline-2 & 5,2 & 4,9 & N.A. & N.A. & 0 & 0 \\
\hline-3 & 0 & 0 & N.A. & N.A. & 0 & 0 \\
\hline-4 & 0 & 0 & N.A. & N.A. & 0 & 0 \\
\hline-5 & 0 & 0 & N.A. & N.A. & 0 & 0 \\
\hline-6 & 0 & 0 & N.A. & N.A. & 0 & 0 \\
\hline & \multicolumn{6}{|c|}{ Response Time (sec) } \\
\hline & \multicolumn{2}{|c|}{ r1 } & \multicolumn{2}{|c|}{ r2 } & \multicolumn{2}{|c|}{ r3b } \\
\hline & $50 \mu \mathrm{M}$ & $100 \mu \mathrm{m}$ & $50 \mu \mathrm{M}$ & $100 \mu \mathrm{M}$ & $50 \mu \mathrm{M}$ & $100 \mu \mathrm{M}$ \\
\hline 2 & 0 & 0 & 30 & 16 & 9,8 & 4,4 \\
\hline 1 & 3100 & 2370 & 255 & 124 & 215 & 123 \\
\hline 0 & 1740 & 980 & 1740 & 980 & 1740 & 980 \\
\hline-1 & 214 & 114 & 27500 & 13000 & 3030 & 2510 \\
\hline-2 & 16,7 & 11,5 & N.A. & N.A. & 0 & 0 \\
\hline-3 & 4,8 & 4,8 & N.A. & N.A. & 0 & 0 \\
\hline-4 & 4 & 4 & N.A. & N.A. & 0 & 0 \\
\hline-5 & 0 & 0 & N.A. & N.A. & 0 & 0 \\
\hline-6 & 0 & 0 & N.A. & N.A. & 0 & 0 \\
\hline
\end{tabular}

(b)

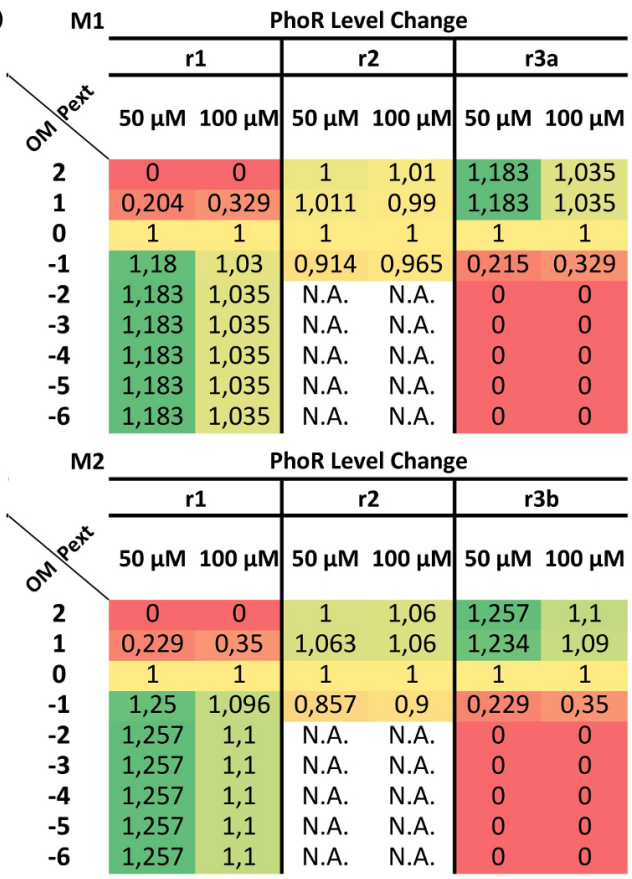

Figure 4: Heatmap displaying the effects of the changes in reaction rates $r 1, r 2, r 3 a$ and $r 3 b$. Each parameter is varied by orders of magnitude $(\mathrm{OM})$ higher and lower. (a) response time and (b) PhoR level change are computed when the system reaches the steady state. For the cases that the system does not have a steady state, response time and PhoR level change are displayed as N.A.. Response time, in seconds, is calculated as the time needed to reach halfway between the initial and final level. The outcome of PhoR level change is normalised with respect to the control model. The analysis is done for Model 1 (M1) and Model 2 (M2) when external $P_{i}$ (Pext) are $50 \mu \mathrm{M}$ and $100 \mu \mathrm{M}$. Red and green represent the decreasing and increasing effects, repectively. Reaction rate $\mathrm{r} 4$ variation is excluded because variations on it imply a departure from the expected system dynamics.

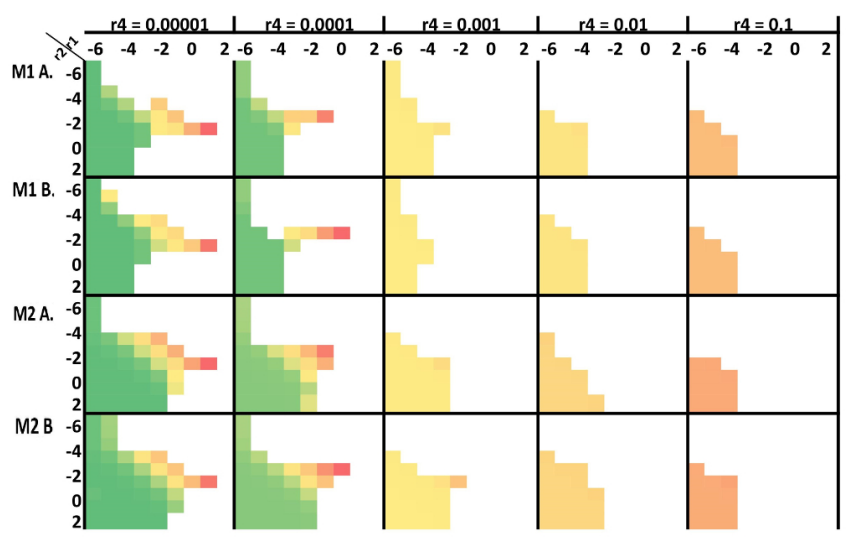

Figure 5: Heatmap displaying the normalised PhoR level change as a result of the variations in $\mathrm{r} 1$ and $\mathrm{r} 2$ together with variations in $\mathrm{r} 4$ rate values. The analysis is performed for Model 1 (M1) and Model 2 (M2) when the external $P_{i}$ concentrations are $50 \mu \mathrm{M}$ (A) and $100 \mu \mathrm{M}$ (B). Rates r1 and r2 are varied by orders of magnitude higher and lower from -6 to 2 . PhoR level change from the beginning to the end of the simulation is computed and displayed. The outcome of PhoR level change is normalized with respect to the control model. Red represents the decreasing effect and green represents the increasing effect.

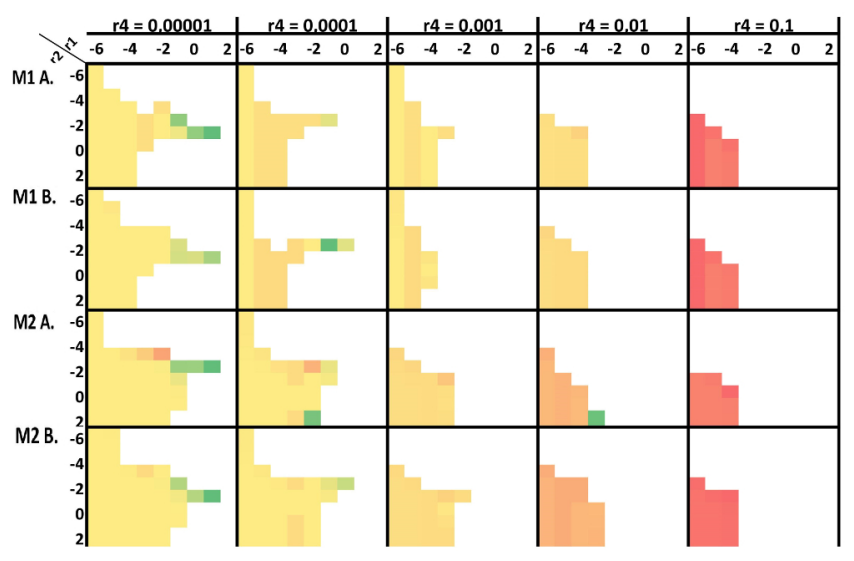

Figure 6: Heatmap displaying the repsonse time, in seconds, as a result of the variations in $\mathrm{r} 1$ and $\mathrm{r} 2$ together with variations in $\mathrm{r} 4$ rate values. The analysis is performed for Model 1 (M1) and Model 2 (M2) when the external $P_{i}$ concentrations are $50 \mu \mathrm{M}(\mathrm{A})$ and $100 \mu \mathrm{M}(\mathrm{B})$. Rates $\mathrm{r} 1$ and $\mathrm{r} 2$ are varied by orders of magnitude higher and lower from -6 to 2. Red represents the decreasing effect and green represents the increasing effect. 
within the alternative models discussed above. In these refined models, reactions 1 and 2 are kept the same as these reactions model the $\mathrm{ABC}$ transporter, which regulates the translocation of $P_{i}$ to the cytosol. We have then considered the interactions of PhoU in the system in accordance with the two models above. As a result of this, we have replaced reactions 3 and 4 with their alternatives that integrate PhoU mechanistically. In this refined model, PhoU interacts with ABC, gets the external $P_{i}$ concentration change signal, and then becomes active (PhoUa).

$$
\begin{aligned}
& \mathrm{ABC}+\mathrm{PhoU} \longrightarrow \text { PhoUa } \\
& \mathrm{PhoUa} \longrightarrow \mathrm{PhoU}
\end{aligned}
$$

After passing to the active state, PhoUa transmits the signal to PhoR, inhibits PhoR activity, and causes the repression of PhoR.

Here, in accordance with the two models above, we have provided alternative reactions to express the affect of PhoU on the system. That is, PhoU can affect PhoR in similar ways as in Model 1 and Model 2.

We first consider the possibility that PhoUa can act like an enzyme, drawing parallels with Model 1.

$$
\begin{aligned}
& \mathrm{PhoR}+\mathrm{PhoUa} \longrightarrow \mathrm{PhoRr}+\mathrm{PhoUa} \\
& \mathrm{PhoRr} \longrightarrow \mathrm{PhoR}
\end{aligned}
$$

Alternatively, we have considered the interaction as in Model 2. In this model, PhoU binds to the histidine kinase PhoR in a reversible manner.

$$
\begin{aligned}
& \text { PhoR }+ \text { PhoUa } \longrightarrow \text { PhoR_PhoUa } \\
& \text { PhoR_PhoUa } \longrightarrow \text { PhoR }+ \text { PhoUa }
\end{aligned}
$$

Figure 7 displays a dynamic representation of these two models with PhoU, which are mathematically described by using the same procedures. PhoU Model 1 includes reactions $1,2,3^{\prime}, 4^{\prime}, 5 a$ and $6 a$ and PhoU Model 2 includes the reactions $1,2,3^{\prime}, 4^{\prime}, 5 b$, and $6 b$. In order to observe the differences that originate from the inclusion of an explicit PhoU mechanism, we have kept the parameter values as before. Therefore, the initial values are kept the same and PhoU initial value is set to $0.22 \mu \mathrm{M}$ as in (Van Dien and Keasling, 1997). The rates of the reactions 1 and 2 are also set to the same values. Reactions $5 a, 6 a$ and $5 b, 6 b$ are assigned the values of the reactions $3 a, 4 a$ and $3 b, 4 b$.

The plots in Figure 7 clearly display the robust adaptation of model with PhoU protein. When the external $P_{i}$ level increases, PhoR concentration decreases in both models. Moreover, we have observed that PhoU Model 1 has a faster response time than PhoU Model 2 for different external $P_{i}$ as shown in Figure 7.

These results provide an analysis of mechanistic interactions of the PhoU, PhoR, and ABC transporter proteins.

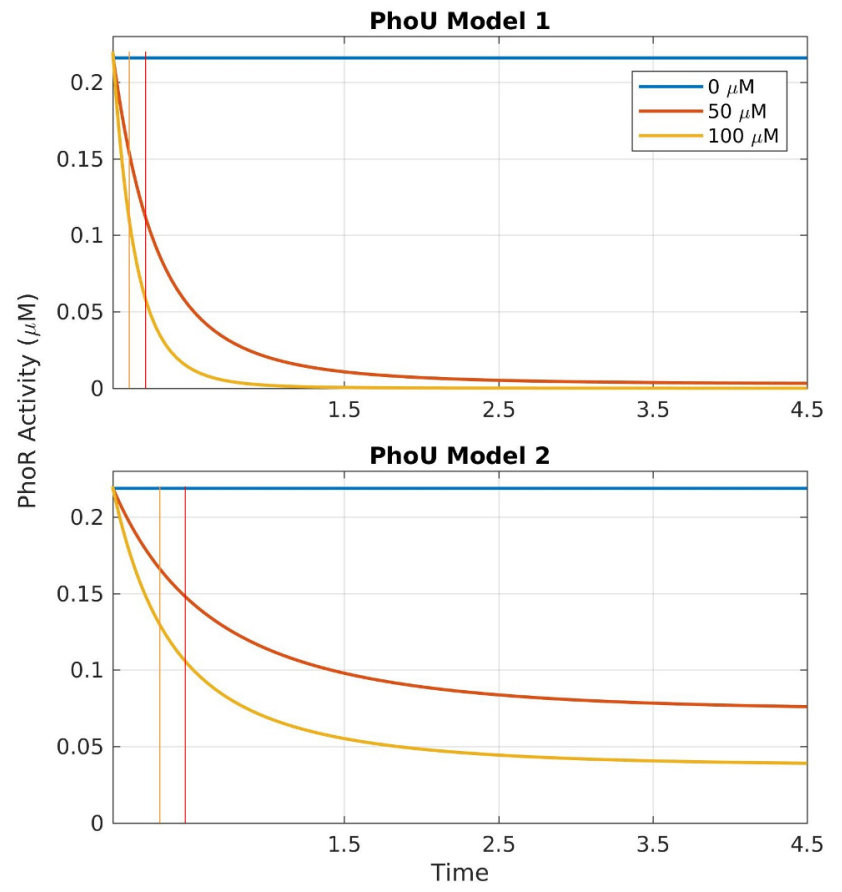

Figure 7: The response of the model with PhoU to variations in the external $P_{i}$ levels. Model 1 includes the reactions 1 , $2,3^{\prime}, 4^{\prime}, 5 a$ and $6 a$, and Model 2 includes $1,2,3^{\prime}, 4^{\prime}, 5 b$ and $6 b$. In all the experiments, the external $P_{i}$ concentrations are 0,50 , and $100 \mu \mathrm{M}$ and a time-course of 4.5 hours is considered. PhoR activity is plotted in blue when $P_{i}$ concentrations is $0 \mu \mathrm{M}$, it is represented with red when external $P_{i}$ concentrations is $50 \mu \mathrm{M}$, and orange color is used when $P_{i}$ concentrations is $100 \mu \mathrm{M}$. Response times are represented with vertical lines. The red line is the response time of dynamics when external $P_{i}$ concentration is $50 \mu \mathrm{M}$ and the orange line is for an external $P_{i}$ concentration of $100 \mu \mathrm{M}$.

The analysis of the results above provides a framework for better understanding $P_{i}$ signal transduction in E. coli. According to our signalling complex model, PhoR is able to sense through PhoU the conformational states of ABC transporter as a consequence of $P_{i}$ transport and then modulate its kinase/phosphatase in accordance with the appropriate response. This model can thus explain the mechanism that provides the adequate response time.

\section{Conclusion}

We have previously reported a quantitative description of the activation of a Pho regulon (Uluşeker et al., 2018, 2017). Here we have developed a model for understanding the role of $\mathrm{ABC}$ transporter and PhoU by an examination of the switch off dynamics. Our model provides descriptions of the possible interaction mechanisms between TCS and ABC transporter and predicts the mechanistic behaviour for dif- 
ferent cases under the conditions of varying external $P_{i}$ concentrations. Understanding the behaviours of TCS requires characterisation of not only the pathway organization, but also the dynamic rates of individual activities in the cell. In addition, by providing testable predictions for the wet lab, the understanding produced from this approach provides insights for future engineering of such biological processes. The model should also allow us to better understand the mechanisms for tuning the regulatory system to be sharper and more rapid. For example variations in the ABC phosphorylation rate can work as an inhibitor for the regulation of PhoR due to the changes in the external $P_{i}$ concentration.

By applying such models to living systems and processes we begin to see mechanistically how an environmental condition produces a change of state in an organism both on the protein function level and gene expression level. It is through such an understanding that the meaning of adaptation and embeddedness in living cells becomes apparent and quantifiable.

\section{Acknowledgements}

This work has been partially funded by the European Union's Horizon 2020 research and innovation programme under the grant agreement No 686585 - LIAR, Living Architecture.

\section{References}

Alon, U. (2007). An introduction to system biology - design principles of biological circuits. Chapman and Hall/CRC.

Alves, R. and Savageau, M. A. (2000). Extending the method of mathematically controlled comparison to include numerical comparisons. Bioinformatics, 16:786-798.

Celani, A. and Vergassola, M. (2009). Bacterial strategies for chemotaxis response. Proc Natl Acad Sci USA, 107:13911396.

Chekabab, S. M., Harel, J., and Dozois, C. M. (1993). Interplay between genetic regulation of phosphate homeostasis and bacterial virulence. Current Opinion in Microbiology, 175:67976809.

Gao, R. and Stock, A. M. (2009). Biological insights from structures of two-component proteins. Annu Rev Microbiol., 63:133-154.

Gardner, S. G., Johns, K. D., Tanner, R., and McCleary, W. R. (2014). The PhoU protein from escherichia coli interacts with PhoR, PstB, and metals to form a phosphate-signaling complex at the membrane. J Bacteriol, 196:1741-1752.

Hsieh, Y. and Wanner, B. L. (2010). Global regulation by the sevencomponent $\mathrm{P}_{i}$ signalig system. Current Opinion in Microbiology, 13:198-203.

Hudek, L., Premachandra, D., Webster, W. A. J., and Bräu, L. (2016). Role of phosphate transport system component PstB 1 in phosphate internalization by nostoc punctiforme. Appl Environ Microbiol, 82:6344-6356.
Lamarche, M. G., Wanner, B. L., Crépin, S., and Harel, J. (2008). The phosphate regulon and bacterial virulence: a regulatory network connecting phosphate homeostasis and pathogenesis. Federation of European Microbiological Societies, 32:461-473.

Peterson, C., Mandel, M. J., and Silhavy, T. J. (2005). Escherichia coli starvation diets: essential nutrients weigh in distinctly. Journal of Bacteriology, 187(22):7549-7553.

Rice, C. D., Pollard, J. E., Lewis, Z. T., and McCleary, W. R. (2009). Employment of a promoter-swapping technique shows that PhoU modulates the activity of the PstCAB2 ABC transporter in Escherichia coli. Applied and Environmental Microbiology, 75:573-582.

Santos-Beneit, F. (2015). The Pho regulon: a huge regulatory network in bacteria. Frontiers in Microbiology, 6:402:1-13.

Shimizu, K. (2014). Regulation systems of bacteria such as Escherichia coli in response to nutrient limitation and environmental stresses. Metabolites, 4:1-35.

Shinar, G., Milo, R., Matínez, M. R., and Alon, U. (2007). Input output robustness in simple bacterial signaling systems. Proc. Natl. Acad. Sci., 104:19931-19935.

Steed, P. M. and Wanner, B. L. (2014). Use of the rep technique for allele replacement to construct mutants with deletions of the PstSCAB-Phou operon: evidence of a new role for the PhoU protein in phosphate regulon. Virulence, 5(8):786-793.

Uluşeker, C., Torres, J., García, J. L., Hanczyc, M. M., Nogales, J., and Kahramanoğulları, O. (2017). A dynamic model of the phosphate response system with synthetic promoters in Escherichia coli. In Proceedings of the European Conference on Artificial Life 2017, pages 412-419. MIT press.

Uluşeker, C., Torres, J., García, J. L., Hanczyc, M. M., Nogales, J., and Kahramanoğulları, O. (2018). Quantifying dynamic mechanisms of auto-regulation in escherichia coli with synthetic promoters in response to varying external phosphate levels. submitted.

Van Dien, S. J. and Keasling, J. D. (1997). A dynamic model of the Escherichia coli phosphate-starvation response. J. Theor. Biol, 190:37-49.

Wanner, B. L. (1996). Phosphorus assimilation and control of the phosphate regulon. In Neidhardt, F., editor, Escherichia coli and Salmonella typhimurium cellular and molecular biology, pages 1357-1381. ASM Press, Washington, D.C.

Wanner, B. L., Jiang, W., Kim, S., Yamagata, S., Haldimann, A., and Daniel, L. L. (1995). Are the multiple signal transduction pathways of the Pho regulon due to cross talk or cross regulation? In Lin, E. and Lynch, A., editors, Regulation of gene expression in Escherichia coli, pages 297-315. R.G. Landes Company, Texas USA. 